\title{
SOME RESULTS ON EXPANSIVE MAPPINGS ${ }^{1}$
}

\author{
RICHARD K. WILLIAMS
}

\begin{abstract}
In this paper, expansive mappings are seen as generalizations of expansive homeomorphisms, and known theorems are generalized. The nonexistence of expansive functions on the open unit interval is proven, and a new example of an expansive homeomorphism on the open unit disk is given. Finally, a method for constructing expansive homeomorphisms from expansive mappings is given.
\end{abstract}

In this paper, we study expansive continuous relations, which we call mappings. These mappings are generalizations of expansive homeomorphisms. It is shown that several of the well-known theorems on expansive homeomorphisms remain valid under the weaker assumptions.

It is proven that there does not exist an expansive function on the open unit interval, and a new example of an expansive homeomorphism on the open unit disk is given.

There also appears in this paper a technique for constructing expansive homeomorphisms from expansive mappings.

If $X$ is a metric space with metric $d$, and if $f$ is a homeomorphism of $X$ onto itself, then $f$ is said to be expansive on $X$ with expansive constant $\delta>0$ if $x, y \in X, x \neq y$, implies $d\left(f^{n}(x), f^{n}(y)\right)>\delta$ for some integer $n$. Distinct points $x$ and $y$ are said to be positively (negatively) asymptotic under $f$ if for each $\epsilon>0$, there is an integer $N$ such that $n>N(n<N)$ implies $d\left(f^{n}(x), f^{n}(y)\right)<\epsilon$. Naturally, $x$ and $y$ are doubly asymptotic if they are both positively and negatively asymptotic.

Let $f$ be a continuous multi-valued transformation of $X$ onto itself. ( $f$ is continuous at $x \in X$ if for each neighborhood $N$ of $f(x)$, there exists $\eta>0$ such that $d(x, y)<\eta$ implies $f(y) \subseteq N$. Henceforth, a continuous multi-valued transformation will simply be called mapping.)

Definition 1. Let $x \in X$. The orbit of $x$ under $f$ is defined by $O(x)$ $=\bigcup_{n=-\infty}^{\infty} f^{n}(x)$.

Definition 2. Let $x \in X$. A suborbit of $x$ under $f$ is a set of the form $\left\{x_{i}: x_{0}=x, x_{i+1} \in f\left(x_{i}\right)\right.$ for each integer $\left.i\right\}$.

Definition 3. $f$ is expansive on $X$ with expansive constant $\delta>0$ if

Received by the editors October 14, 1969.

AMS 1969 subject classifications. Primary 5482; Secondary 5460, 5465.

Key words and phrases. Expansive function, expansive mapping, expansive homeomorphism, orbit, iterates, asymptotic points.

1 This research was supported in part by NASA Grant 44-007-006. 
$x, y \in X, x \neq y$ implies for each suborbit $A$ of $x$ and for each suborbit $B$ of $y$, there exist $x_{n} \in A, y_{n} \in B$ such that $d\left(x_{n}, y_{n}\right)>\delta$.

DEFINITION 4. $x$ and $y$ are positively (negatively) asymptotic under $f$ if $x \neq y$ and if for each $\epsilon>0$, there is an integer $N$ such that $n>N$ $(n<N)$ implies

$$
\inf \left\{d(a, b): a \in f^{n}(x), b \in f^{n}(y)\right\}<\epsilon .
$$

It is clear that these definitions reduce to the standard ones when $f$ is a homeomorphism.

Henceforth, unless stated otherwise, we will assume that $X$ is compact, and that $f$ is a mapping of $X$ onto itself.

Let $S(X)$ be the set of all single-valued transformations from the in tegers into $X$. For each $z \in S(X)$, denote $z(i)$ by $z_{i}$. Let $\epsilon$ be a positive number. For $z, w \in S(X)$, define

$$
\rho(z, w)=d\left(z_{0}, w_{0}\right)+\epsilon \sum_{i \neq 0} \frac{d\left(z_{i}, w_{i}\right)}{2^{|i|}} .
$$

Then it is readily verified that $\rho$ is a metric for $S(X)$. Call the resulting space $S(X, \epsilon)$.

Let $S(X, f, \epsilon)=\left\{z \in S(X, \epsilon): z_{i+1} \in f\left(z_{i}\right)\right.$ for each $\left.i\right\}$, and let $h$ be the transformation of $S(X, \epsilon)$ on to itself defined by $(h(z))_{i}=z_{i+1}$ for each $i$. (Thus $h$ is the "shift" transformation.)

The spaces $S(X, \epsilon)$ and $S(X, f, \epsilon)$ and the transformation $h$ have the following properties:

(1) The product and metric topologies for $S(X)$ are equivalent.

(2) $S(X, f, \epsilon)$ is a closed subset of $S(X, \epsilon)$, and is therefore compact.

(3) $h$ is a homeomorphism of $S(X, f, \epsilon)$ on to itself.

(4) $h$ is an expansive homeomorphism on $S(X, f, \epsilon)$ if and only if $f$ is an expansive mapping on $X$.

(5) If $X$ is homeomorphic to $Y$ under $g$, then $S(X, f, \epsilon)$ is homeomorphic to $S\left(Y, g g^{-1}, \epsilon\right)$ under the transformation $H$ defined by $(H(z))_{i}=g\left(z_{i}\right)$. Also, $h_{2}=H h_{1} H^{-1}$, where $h_{1}$ and $h_{2}$ are the shift transformations on $S(X, f, \epsilon)$ and $S\left(Y, g f g^{-1}, \epsilon\right)$, respectively.

(6) If $z, w \in S(X, f, \epsilon)$, if $z_{i} \neq w_{i}$, and if $z$ and $w$ are positively (negatively) asymptotic under $h$, then $z_{i}$ and $w_{i}$ are positively (negatively) asymptotic under $f$.

The proofs of properties (1), (2), (3), and (5) are straightforward, and will be omitted.

Proof of (4). Let $z, w \in S(X, f, \epsilon), z \neq w$, and suppose that $f$ is an expansive mapping on $X$ with expansive constant $\delta$. Since $z \neq w$, then $z_{n} \neq w_{n}$ for some $n$. It is clear that the set of $z_{i}$ 's form a suborbit of 
$z_{n}$ and the set of $w_{i}$ 's form a suborbit of $w_{n}$. Hence, there exists $m$ such that $d\left(z_{m+n}, w_{m+n}\right)>\delta$, and so

$$
\rho\left(h^{m+n}(z), h^{m+n}(w)\right) \geqq d\left(z_{m+n}, w_{m+n}\right)>\delta,
$$

and $h$ is expansive with expansive constant $\delta$.

Now suppose that $h$ is expansive with expansive constant $\delta$. Suppose that $\delta /(1+2 \epsilon)$ is not an expansive constant for $f$. Then there exist $z_{0}, w_{0} \in X, z_{0} \neq w_{0}$, such that for some suborbit $A$ of $z_{0}$ and some suborbit $B$ of $w_{0}, d\left(z_{n}, w_{n}\right) \leqq \delta /(1+2 \epsilon)$ for each $z_{n} \in A, w_{n} \in B$. The $z_{n}$ 's and $w_{n}$ 's define points in $S(X, f, \epsilon)$ in the natural way. It is clear that $z \neq w$, and if $k$ is any integer,

$$
\rho\left(h^{k}(z), h^{k}(w)\right) \leqq \frac{\delta}{1+2 \epsilon}\left(1+\epsilon \sum_{i \neq 0} \frac{1}{2^{|i|}}\right)=\delta,
$$

a contradiction.

PROOF OF (6).

$\inf \left\{d(a, b): a \in f^{n}\left(z_{i}\right), b \in f^{n}\left(w_{i}\right)\right\} \leqq d\left(z_{n+i}, w_{n+i}\right) \leqq \rho\left(h^{n+i}(z), h^{n+i}(w)\right) ;$

the property follows from this inequality.

Each of the next five theorems is a generalization of a theorem in the literature. In all of these, we assume that $f$ is an expansive mapping on $X$ with expansive constant $\delta$.

THEOREM 1. If $g$ is a homeomorphism of $X$ onto a space $Y$, then $g f g^{-1}$ is an expansive mapping on $Y$.

Proof. Since $f$ is an expansive mapping on $X, h_{1}$ is an expansive homeomorphism on $S(X, f, \epsilon)$ so, $H h_{1} H^{-1}=h_{2}$ is an expansive homeomorphism on $S\left(Y, g f g^{-1}, \epsilon\right)\left[2\right.$, Theorem 1], and so $g f g^{-1}$ is an expansive mapping on $Y$.

THEOREM 2. If $X$ is infinite, there exist $a, b, c, d \in X$ such that $a$ and $b$ are positively asymptotic under $f$ and $c$ and $d$ are negatively asymptotic under $f$.

Proof. There exist $z, w \in S(X, f, \epsilon)$ such that $z$ and $w$ are positively asymptotic under $h\left[3\right.$, Theorem 2]. Then $z_{i} \neq w_{i}$ for some $i$, and by property (6), $z_{i}$ and $w_{i}$ are positively asymptotic under $f$. The second half of the theorem follows similarly.

THEOREM 3. Let

$$
\theta=\sup \{\eta: \eta \text { is an expansive constant for } f\} .
$$

Then $\theta$ is not an expansive constant for $f$. 
Proof. Let

$$
\theta_{i}=\sup \{\eta: \eta \text { is an expansive constant for } h \text { on } S(X, f, 1 / i)\},
$$

$i=1,2, \cdots$. Let $M=\operatorname{diam}(X)$, and let $\alpha>0$. There exist $z_{0}, w_{0} \in X$, $z_{0} \neq w_{0}$, a suborbit $A$ of $z_{0}$, and a suborbit $B$ of $w_{0}$ such that $z_{k} \in A$, $w_{k} \in B$ implies $d\left(z_{k}, w_{k}\right) \leqq \theta+\alpha$. Define $z, w \in S(X, f, 1 / i)$ in the natural way from the $z_{k}$ 's and the $w_{k}$ 's. Then

$$
\rho\left(h^{n}(z), h^{n}(w)\right)=d\left(z_{n}, w_{n}\right)+\frac{1}{i} \sum_{j \neq 0} \frac{d\left(z_{n+j}, w_{n+j}\right)}{2^{|j|}} \leqq \theta+\alpha+\frac{2 M}{i}
$$

for each $n$. Thus $\theta_{i} \leqq \theta+\alpha+2 M / i$. Since $\alpha$ was arbitrary, $\theta_{i} \leqq \theta+2 M / i$.

There exist distinct points in $S(X, f, 1 / i)$, say $s^{i}$ and $t^{i}$, such that $d\left(s_{n}^{i}, t_{n}^{i}\right) \leqq \rho\left(h^{n}\left(s^{i}\right), h^{n}\left(t^{i}\right)\right) \leqq \theta_{i}$ for each $n$, since $\theta_{i}$ is not an expansive constant for $h$ on $S(X, f, 1 / i)$ [2, Theorem 6]. Since $\theta / 2$ is an expansive constant for $f$, there is an integer $m$ such that $d\left(s_{m}^{i}, t_{m}^{i}\right)>\theta / 2$. (The coordinates of $s^{i}$ and $t^{i}$ form suborbits.) Let $u^{i}=h^{m}\left(s^{i}\right)$ and $v^{i}=h^{m}\left(t^{i}\right)$. Then $d\left(u_{n}^{i}, v_{n}^{i}\right) \leqq \theta_{i}$ for each $n$, and $\rho\left(u^{i}, v^{i}\right) \geqq d\left(u_{0}^{i}, v_{0}^{i}\right)>\theta / 2$. We may assume that for each fixed $n, u_{n}^{i}$ converges to some $u_{n} \in X$, and $v_{n}^{i}$ converges to some $v_{n} \in X$. Clearly $d\left(u_{0}, v_{0}\right) \geqq \theta / 2>0$, and $d\left(u_{n}, v_{n}\right) \leqq \theta$, since $d\left(u_{n}^{i}, v_{n}^{i}\right) \leqq \theta_{i} \leqq \theta+2 M / i$ for each $i$. Also $u_{n} \in f^{n}\left(u_{0}\right)$, $v_{n} \in f^{n}\left(v_{0}\right)$, by the continuity of $f[1$, p. 111, Theorem 4]. Since the set of $u_{n}$ 's is a suborbit of $u_{0}$ and the set of $v_{n}$ 's is a suborbit of $v_{0}$, and $d\left(u_{n}, v_{n}\right) \leqq \theta$ for each $n, \theta$ is not an expansive constant for $f$.

Theorem 4. Let $x \neq y$. Suppose there is a suborbit $A$ of $x$ and $a$ suborbit $B$ of $y$ and an integer $N$ such that $n>N(n<N)$ implies $d\left(x_{n}, y_{n}\right)$ $\leqq \delta$. Then $x$ and $y$ are positively (negatively) asymptotic.

Proof. Choose $\delta^{\prime}>\delta$ such that $\delta^{\prime}$ is an expansive constant for $f$. Again, let $M=\operatorname{diam}(X)$, and choose a positive integer $i$ such that $2 M / i<\delta^{\prime}-\delta$. Define $z, w \in S(X, f, 1 / i)$ in the natural way from the $x_{n}$ 's in $A$ and the $y_{n}$ 's in $B$, i.e. let $z_{n}=x_{n}, w_{n}=y_{n}$ for each $n$. Now $n>N(n<N)$ implies $\rho\left(h^{n}(z), h^{n}(w)\right)<\delta^{\prime}$, so since $\delta^{\prime}$ is an expansive constant for $h$ on $S(X, f, 1 / i), z$ and $w$ are positively (negatively) asymptotic under $h[3$, Lemma 1$]$. By property (6), $z_{0}$ and $w_{0}$, i.e. $x$ and $y$, are positively (negatively) asymptotic.

TheOREM 5. If $X$ is infinite, then $m \neq n$ implies

$$
\sup \left\{\sup \left\{d(a, b): a \in f^{m}(x), b \in f^{n}(x)\right\}: x \in X\right\}>\delta .
$$

Proof. Choose $\delta^{\prime}$ and $i$ as in the proof of the preceding theorem. Since $\delta^{\prime}$ is an expansive constant for $h$ on $S(X, f, 1 / i)$, there exists 
$z \in S(X, f, 1 / i)$ such that $\rho\left(h^{m}(z), h^{n}(z)\right)>\delta^{\prime}[2$, Theorem 2]. Thus $d\left(z_{m}, z_{n}\right)>\delta$, and

$$
\sup \left\{\sup \left\{d(a, b): a \in f^{m}(x), b \in f^{n}(x)\right\}: x \in X\right\}>\delta, \quad \text { q.e.d. }
$$

One might think that this theorem could be strengthened to say

$$
\sup \left\{\inf \left\{d(a, b): a \in f^{m}(x), b \in f^{n}(x)\right\}: x \in X\right\}>\delta,
$$

but this is not so. For let $X$ be the unit circle in the complex plane, and let $f(x)=x^{2}$. Then $f$ is expansive with expansive constant $\sqrt{ } 2$. (If $x_{1} \neq x_{2}$, there is a positive integer $j$ such that $\pi / 2<\mid \arg \left(f^{j}\left(x_{1}\right)\right.$ ) $-\arg \left(f^{j}\left(x_{2}\right)\right) \mid \leqq \pi$, so $\left|f^{j}\left(x_{1}\right)-f^{j}\left(x_{2}\right)\right|>\sqrt{ } 2$.) Choose positive integers $m$ and $n$ such that $m \neq n, 2 \pi / 2^{m}<\pi / 4$, and $2 \pi / 2^{n}<\pi / 4$. Each $x \in X$ is of the form $e^{i \theta}, 0 \leqq \theta<2 \pi$. Then $f^{-m}(x)$ contains $e^{i \theta / 2^{m}}$, and $0 \leqq \theta / 2^{m}<\pi / 4$. A similar statement holds for $f^{-n}(x)$. Thus,

$$
\inf \left\{|a-b|: a \in f^{-m}(x), b \in f^{-n}(x)\right\}<(2-\sqrt{ } 2)^{1 / 2}
$$

for each $x \in X$, so that

$$
\sup \left\{\inf \left\{|a-b|: a \in f^{-m}(x), b \in f^{-n}(x)\right\}: x \in X\right\} \leqq(2-\sqrt{ } 2)^{1 / 2}<\sqrt{ } 2 .
$$

CoRollaRY. Let $\Omega$ be a bounded open connected subset of the plane. Let $g$ be a function which is analytic on $\Omega$, and let $g(\Omega) \subseteq \Omega$. Let $C$ be a compact subset of $\Omega$, and let $g(C)=C$. Then $g$ is not expansive on $C$.

Proof. Consider $\left\{g^{n}: n\right.$ is a positive integer $\}$. Since this is a normal family of analytic functions, there is a subsequence $\left\{n_{j}\right\}$ such that $\left\{f^{n_{j}}\right\}$ converges uniformly on $C$. Suppose $f$ is expansive on $C$ with expansive constant $\delta$. There exists a positive integer $N$ such that $n_{j}, n_{k}>N$ implies $\left|f^{n_{j}}(z)-f^{n_{k}}(z)\right|<\delta$ for each $z \in C$. Choose $j \neq k$ such that $n_{j}, n_{k}>N$. By the theorem above, there is a point $w \in C$ such that $\left|f^{n_{i}}(w)-f^{n_{k}}(w)\right|>\delta$. We thus have a contradiction, and the theorem is proved.

Most of the other standard theorems on expansive homeomorphisms generalize in this setting. (See [6].) For example, $f$ is expansive if and only if $f^{n}$ is expansive for $n \neq 0$. (See [5, Theorem 2.2].)

In [7], the author showed that there does not exist an expansive function on the closed unit interval, and an example of an expansive mapping on this interval was given. We now prove the following related result:

THEOREM 6. Let $f$ be a function mapping the open unit interval onto itself. Then $f$ is not expansive.

Proof. If $f$ is one-to-one, the result is known. (See [2].) Also, if $f$ 
is uniformly continuous on $(0,1)$, the proof of Theorem 2 of [7] can easily be modified to yield the desired result. And if $f$ has at least two fixed points, the above mentioned modification yields the result. Thus we may assume that $\lim \sup _{x \rightarrow 0} f(x)=1, \lim _{\inf _{x \rightarrow 1}} f(x)=0$, and that at least one of $\lim _{x \rightarrow 0} f(x)$ and $\lim _{x \rightarrow 1} f(x)$ does not exist.

Suppose that $\lim _{x \rightarrow 0} f(x)$ does not exist. (The other case is similar and will be omitted.) Let $\delta>0$. Choose $\eta$ such that $0<\eta<\delta$ and $\lim \inf _{x \rightarrow 0} \leqq 1-\eta$. There exist $a, b \in(0, \eta)$ and $a_{-1}, b_{-1} \in(1-\eta, 1)$ such that $f(a)=f(b), f\left(a_{-1}\right)=a$, and $f\left(b_{-1}\right)=b$. Also, there exist $a_{-2}, b_{-2}$ $\in(0, \eta)$ such that $f\left(a_{-2}\right)=a_{-1}$ and $f\left(b_{-2}\right)=b_{-1}$. Continuing this process, we can define orbits $A$ and $B$ of $a$ and $b$ respectively, and $a_{n} \in A$, $b_{n} \in B$ implies $d\left(a_{n}, b_{n}\right)<\eta<\delta$ for each integer $n$. Thus $\delta$ is not an expansive constant for $f$. Since $\delta$ was arbitrary, the proof is complete.

COROLlARY. The property of admitting expansive functions is not a topological property.

Proof. It is clear that $f(x)=2 x$ is expansive on the reals. In [4], Reddy showed that there do exist expansive functions on the open unit disk; in fact, the function can be taken to be a homeomorphism. In the author's opinion, the example that follows is somewhat simpler than that of Reddy.

Before giving the example, we need some preliminaries. For $\alpha \in[0,2 \pi)$, let $S_{\alpha}$ be the spiral whose polar equation is $r$ $=(\theta-\alpha) /(1+\theta-\alpha), r \geqq 0$.

LEммA. The spirals defined above are disjoint except at the origin, and their union covers the open unit disk.

Proof. Clearly the origin is common to each spiral. Let $(\alpha, \theta)$ denote the point on $S_{\alpha}$ with corresponding angle $\theta$. If $\left(\alpha_{1}, \theta_{1}\right)=\left(\alpha_{2}, \theta_{2}\right)$, then since $\theta-\alpha=r /(1-r), \theta_{1}-\alpha_{1}=\theta_{2}-\alpha_{2}$ and $\theta_{1} \equiv \theta_{2}(\bmod 2 \pi)$. Hence $\alpha_{1}-\alpha_{2}=\theta_{1}-\theta_{2}=2 k \pi$. Since $\alpha_{1}, \alpha_{2} \in[0,2 \pi), k=0$, and so $\alpha_{1}=\alpha_{2}$, $\theta_{1}=\theta_{2}$. Next, suppose we are given a point with polar coordinates $r$ and $\theta$, where $r<1$. Choose an integer $n$ such that $\theta+2 n \pi-r /(1-r)$ $\in[0,2 \pi)$. Defining $\alpha$ to be $\theta+2 n \pi-r /(1-r)$, we see that our given point can be represented as $(\alpha, \theta+2 n \pi)$. Hence the spirals cover the disk.

Let $f$ be defined as the function which maps $(\alpha, \theta)$ to $(\alpha, 2 \theta+r)$ if $0 \leqq \alpha \leqq 2 \pi r$, and to $(\alpha, 2 \theta-(\alpha-2 \pi r) /(1-r)+r)$ if $2 \pi r \leqq \alpha<2 \pi$. Then we have the following theorem.

THEOREM 7. The function $f$ defined above is a positively expansive homeomorphism of the open unit disk onto itself. 
Proof. Let the image of $(\alpha, \theta)$ under $f$ be denoted by $(\alpha, \theta)^{\prime}$. Straightforward calculations and the facts that $\theta \geqq \alpha$ and $\alpha<2 \pi$ show that if $(\alpha, \theta)^{\prime}=\left(\alpha, \theta^{\prime}\right)$, then $\theta^{\prime} \geqq \theta$. This shows that each spiral maps on to itself, and thus the mapping is onto. It is also easy to show that in all cases, if $\left(\alpha, \theta_{1}\right)^{\prime}=\left(\alpha, \theta_{1}^{\prime}\right)$ and $\left(\alpha, \theta_{2}\right)^{\prime}=\left(\alpha, \theta_{2}^{\prime}\right)$, where $\theta_{1}<\theta_{2}$, then $\theta_{1}{ }^{\prime}<\theta_{2}{ }^{\prime}$. Hence the mapping is one-to-one.

Next, we show that $f$ is continuous. Note that since $\theta-\alpha=r /(1-r)$, $\left(\alpha_{n}, \theta_{n}\right) \rightarrow\left(\alpha_{0}, \theta_{0}\right)$ if and only if $\theta_{n}-\alpha_{n} \rightarrow \theta_{0}-\alpha_{0}$ and $\theta_{n} \rightarrow \theta_{0}(\bmod 2 \pi)$. Since each $\alpha \in[0,2 \pi)$, this condition is equivalent to either $\alpha_{n} \rightarrow \alpha_{0}$ and $\theta_{n} \rightarrow \theta_{0}$ or $\alpha_{n} \rightarrow 2 \pi, \alpha_{0}=0$, and $\theta_{n} \rightarrow \theta_{0}+2 \pi$. From these observations, we see that in all cases, $\left(\alpha_{n}, \theta_{n}\right) \rightarrow\left(\alpha_{0}, \theta_{0}\right)$ implies $\left(\alpha_{n}, \theta_{n}\right)^{\prime}$ $\rightarrow\left(\alpha_{0}, \theta_{0}\right)^{\prime}$, i.e. $f$ is continuous on the open disk. It follows that $f$ is a homeomorphism of the open disk on to itself.

We now show that $f$ is positively expansive. Let $\delta=\frac{1}{2}$. (Actually any $\delta<1$ will do.) A simple calculation shows that if $2 \pi r \leqq \alpha<2 \pi$, if $(\alpha, \theta)^{\prime}=\left(\alpha, \theta^{\prime}\right)$, and if $\alpha<\theta$ (i.e. $(\alpha, \theta)$ is not the origin), then $\theta^{\prime}>2 \theta$ $-\alpha$. If $\left(\alpha, \theta^{(n)}\right)$ denotes the $n$th positive iterate of $(\alpha, \theta)$, and if $r^{(n)}=\left(\theta^{(n)}-\alpha\right) /\left(1+\theta^{(n)}-\alpha\right)$, then we see that if $n$ is sufficiently large, $0 \leqq \alpha \leqq 2 \pi r^{(n)}$. It is now clear that the positive iterates of each point other than the origin tend to the boundary of the disk.

Let $\left(\alpha_{1}, \theta_{1}\right) \neq\left(\alpha_{2}, \theta_{2}\right)$. If one of the points is the origin, then clearly positive iterates of the two points eventually get greater than $\delta$ apart. Otherwise, we may assume that $r_{1}, r_{2}>\frac{1}{2}$ and that $\alpha_{1} \leqq 2 \pi r_{1}$, $\alpha_{2} \leqq 2 \pi r_{2}$. (If not, perform an appropriate number of preliminary positive iterations.) If $\alpha_{1}=\alpha_{2}$, it is clear that for sufficiently large $n$, $\theta_{1}{ }^{(n)}$ and $\theta_{2}{ }^{(n)}$ (reduced, $\bmod 2 \pi$ ) differ by more than $\pi / 2$ and no more than $\pi$, and therefore $\left(\alpha_{1}, \theta_{1}{ }^{(n)}\right)$ and $\left(\alpha_{2}, \theta_{2}{ }^{(n)}\right)$ are more than $\delta$ apart.

Finally, we may suppose than $\alpha_{1} \neq \alpha_{2}, r_{1}, r_{2}>\frac{1}{2}, \alpha_{1} \leqq 2 \pi r_{1}, \alpha_{2} \leqq 2 \pi r_{2}$. If $\theta_{1} \neq \theta_{2}$, then positive iterates of $\left(\alpha_{1}, \theta_{1}\right)$ and $\left(\alpha_{2}, \theta_{2}\right)$ get more than $\delta$ apart as in the preceding case. If $\theta_{1}=\theta_{2}$, then $\theta_{1}{ }^{\prime}=2 \theta_{1}+r_{1}$ and $\theta_{2}{ }^{\prime}$ $=2 \theta_{2}+r_{2}$, and so $\theta_{1}{ }^{\prime} \neq \theta_{2}{ }^{\prime}$, since $r_{1} \neq r_{2}$. Now, the preceding proof can be applied, completing the verification that $f$ is positively expansive.

We close with several examples. In [7], the author gave an example of an expansive mapping on $[0,1]$. This mapping was not a function. If we call this mapping $f$, then the mapping defined by $\left(x_{1}, x_{2}, \cdots, x_{n}\right) \rightarrow\left(f\left(x_{1}\right), f\left(x_{2}\right), \cdots, f\left(x_{n}\right)\right)$ is clearly an expansive mapping on the closed $n$-cube. Using Theorem 1 , we obtain an expansive mapping on the closed $n$-cell. If we call the closed $n$-cell $X$ and the corresponding mapping $F$, then the "shift" is an expansive homeomorphism on $S(X, F, 1)$ by property (4).

Next, let $X$ be the family of functions which are analytic for $z \neq 0$, and which can be written in the form $\sum_{n=-1}^{\infty} a_{n} z^{n}, a_{0}$ an integer. 
Define a metric $\rho$ on $X$ by $\rho(f, g)=\sup _{|z|=1}|f(z)-g(z)|$. Let $F$ be defined on $X$ by $F(f(z))=\{g(z) \in X: g(z)-f(z / 2)$ is an integer $\}$. Thus, $F$ is not a function, but a relation which maps $X$ onto itself. Clearly $F$ will be continuous if the transformation $f(z) \rightarrow f(z / 2)$ is continuous.

Let $\epsilon>0$. Let

$$
f(z)=\sum_{n=-1}^{\infty} a_{n} z^{n}, \quad g(z)=\sum_{n=-1}^{\infty} b_{n} z^{n}, \quad \rho(f, g)<\epsilon / 2 .
$$

Then

$$
\begin{aligned}
& \rho\left(f\left(\frac{z}{2}\right), g\left(\frac{z}{2}\right)\right) \\
& =\sup _{|z|=1}\left|\sum_{n=-1}^{\infty}\left(a_{n}-b_{n}\right)\left(\frac{z}{2}\right)^{n}\right|=\sup _{|z|=1}|z|\left|\sum_{n=-1}^{\infty}\left(a_{n}-b_{n}\right)\left(\frac{z}{2}\right)^{n}\right| \\
& =2 \sup _{|z|=1}\left|\sum_{n=-1}^{\infty}\left(a_{n}-b_{n}\right)\left(\frac{z}{2}\right)^{n+1}\right| \leqq 2 \sup _{|z|=1}\left|\sum_{n=-1}^{\infty}\left(a_{n}-b_{n}\right) z^{n+1}\right| \\
& =2 \sup _{|z|=1}|z|\left|\sum_{n=-1}^{\infty}\left(a_{n}-b_{n}\right) z^{n}\right|=2 \sup _{|z|=1}\left|\sum_{n=-1}^{\infty}\left(a_{n}-b_{n}\right) z^{n}\right| \\
& =2 \rho(f, g)<\epsilon .
\end{aligned}
$$

(The inequality above is of course justified by the maximum modulus theorem.) Thus $F$ is a mapping on $X$.

We now show that $F$ is expansive on $X$. Let $f(z)=\sum_{n=-1}^{\infty} a_{n} z^{n}$, $g(z)=\sum_{n=-1}^{\infty} b_{n} z^{n}, f \neq g$. Let $A$ and $B$ be orbits of $f$ and $g$ respectively. Suppose there exist $f_{k} \in A, g_{k} \in B$ such that $f_{k}$ and $g_{k}$ have different constant terms in their expansions. If these constant terms are $a$ and $b$, then it follows from the usual integral representation for the coefficients in a Laurent expansion that $|a-b| \leqq \rho\left(f_{k}, g_{k}\right)$. Since $a$ and $b$ are distinct integers, $\rho\left(f_{k}, g_{k}\right) \geqq 1$. We may thus assume that for each $k, f_{k}$ and $g_{k}$ have the same constant terms. Then

$$
\rho\left(f_{k}, g_{k}\right)=\sup _{|z|=1}\left|2^{k} \frac{a_{-1}-b_{-1}}{z}+\sum_{n=1}^{\infty}\left(a_{n}-b_{n}\right)\left(\frac{z}{2^{k}}\right)^{n}\right| .
$$

If $a_{-1} \neq b_{-1}$, then $\lim _{k \rightarrow \infty} \rho\left(f_{k}, g_{k}\right)=\infty$. If $a_{-1}=b_{-1}$, then $\lim _{k \rightarrow-\infty} \rho\left(f_{k}, g_{k}\right)$ $=\infty$, the second limit being justified by the maximum modulus theorem and Liouville's theorem. Thus, if $0<\delta<1, \delta$ is an expansive constant for $F$ on $X$. $F$ is not positively expansive, as is seen by tak- 
ing $f(z)=0$ and $g(z)=\delta z$. $F$ is also not negatively expansive, as is seen by taking $f(z)=0, g(z)=\delta / z$.

The author wishes to thank the referee for several helpful suggestions. In particular, the referee pointed out that the example of the expansive mapping on $[0,1]$ mentioned above leads to an expansive homeomorphism on a compact space with trivial Cech homology.

\section{REFERENCES}

1. Claude Berge, Topological spaces, Oliver and Boyd, London, 1963.

2. B. F. Bryant, Expansive self-homeomorphisms of a compact metric space, Amer. Math. Monthly 69 (1962).

3. - On expansive homeomorphisms, Pacific J. Math. 10 (1960), 1163-1167. MR $22 \# 11382$.

4. William Reddy, The existence of expansive homeomorphisms on manifolds, Duke Math. J. 32 (1965), 627-632. MR 32 \#4679.

5. W. R. Utz, Unstable homeomorphisms, Proc. Amer. Math. Soc. 1 (1950), 769 774.

6. Richard K. Williams, Doctoral Thesis, Vanderbilt University, Nashville, Tenn., 1965.

7. - A note on expansive mappings, Proc. Amer. Math. Soc. 22 (1969), 145147.

Southern Methodist University, Dallas, Texas 75222 